\title{
Identification of Abnormal Human Behavior in Intelligent Video Surveillance System
}

\author{
Bo Zhai \\ College of computer and Communication Engineering, beijing information technology collge, information \\ processing technology, BeiJing,100018
}

Keywords: KL distance similarity, abnormal population, attribute label, preference distribution model

\begin{abstract}
Focusing on the security issues of current group activities, strengthening the prevention of group incidents is the focus of current thinking. Combined with the above requirements, crowd abnormality recognition algorithms have begun to enter people's field of vision and are valued. In this regard, this paper combines crowd abnormality and intelligent monitoring video acquisition principles, proposes a crowd recognition algorithm based on preference distribution model, uses KL distance similarity to complete the labeling of common attribute labels, and then uses the preference distribution model to complete the classification of abnormal behavior, and get the judgment of abnormal behavior. Finally, through the test verification method, the verification of the above model is completed and its feasibility is proved.
\end{abstract}

With the continuous increase of modern intelligent monitoring, video images show a geometric growth rate. How to combine these intelligent monitoring images to complete the detection of abnormal behaviors among them and provide references and reference for the current group safety management is the focus of current thinking and research. Video surveillance is an important part of security work. The traditional video surveillance system mainly uses the on-duty personnel to manually identify and analyze the scene images displayed by the video, in order to find abnormal and suspicious information on the screen. Obviously, the efficiency of this type of video surveillance recognition detection method is extremely low, and it is only possible to adopt video playback methods afterwards, making it difficult to realize real-time monitoring and occupying a large amount of time and manpower. In addition, the concentration of attention of personnel on duty is greatly affected by subjective factors such as the degree of fatigue, which can easily lead to incorrect judgments, resulting in misstatements, omissions, and other consequences. In summary, the traditional video surveillance system has great limitations in terms of the timeliness and accuracy of the alarm, and it is difficult to meet the needs of real-time monitoring and analysis, and lacks the capability of timely and accurate alarming and rapid emergency response. With the continuous advancement of science and technology, intelligent video surveillance technology has made great progress in recent years. By using computers instead of traditional labor to identify, track, and analyze video images, automatic monitoring of abnormal and suspicious information is realized, Can quickly and accurately carry out alarm and emergency response processing, so as to play a more powerful security role.

\section{Abnormal Population Characteristics}

The crowd is the collection of human individuals. Its scene types include both structured and unstructured. The positioning of abnormalities in the crowd is a prerequisite for identifying abnormal situations. Abnormality is abnormal. The so-called anomaly refers to situations that are contrary to normal norms of social civilization and behavioral habits, and are often harmful. When abnormalities occur in the population, they can be classified as abnormal population types. The abnormal population characteristics analyze and summarize the characteristics of the population in abnormal events. It can be found that the main characteristics of the abnormal population include the following two aspects: 
(1) Abnormal characteristics of crowd density. Population density is a representative population characteristic that reflects various anomalous events. Its abnormal characteristics include population density exceeding the threshold and abnormal events. Many existing crowd identification algorithms are based on crowd density characteristics.

(2) The abnormal characteristics of crowd movement patterns. The characteristics of the crowd's movement pattern include the speed of the crowd and the direction of movement of the crowd. Among them, the characteristics of crowd movement speed include kinetic energy, motion entropy, etc., while the crowd movement direction characteristics include the direction of motion histogram and the direction of probability distribution.

\section{Intelligent Video Surveillance System}

An intelligent video surveillance system is an intelligent technology capable of identifying people and objects, and is mainly applied in the fields of face recognition, license plate recognition, and object running track identification. In the application process, the intelligent video surveillance system mainly relies on intelligent technologies such as image processing and pattern recognition to interpret and respond to key information in the video. The research object that the intelligent video monitoring system faces is all the moving objects in the video. The specific recognition steps are divided into three steps: First, identify all the moving objects in the video to obtain the motion characteristics of each moving object, such as Shape, color, and movement methods, Secondly, it records the position, moving speed and trajectory of the moving object in the video, and provides a reference for people to identify and identify the moving target. Finally, it judges the abnormal behavior in the moving target and makes relevant early warnings and decisions. At present, the most commonly used intelligent video surveillance system is mainly composed of four major modules, which are image acquisition, processing,

\section{Crowd Abnormal Behavior Recognition Based on Preference Distribution Learning}

Usually, abnormal behavior is generally continuous and variable at the time of occurrence. For example, in the event of a crowd panicking to escape such anomalous behavior, it is highly probable that people will fall, which will lead to stampede or other more serious abnormal behavior. Therefore, for the abnormal behavior identification of the crowd, a single behavior label cannot accurately mark the longer behavior sequence. At present, the most commonly used longer behavior sequence is labeled as a multiple tag ranking method. The method mainly includes two major tasks in the application process, namely multi-tag classification and multi-tag sorting. The responsibility of the multi-label classification is to divide the target sequence into two categories, related behavior labels and unrelated behavior labels, and then sort them by multi-label sorting. Although this method can realize the marking of longer behavior sequences, its preference in the actual application process is more subjective, so that the final recognition result varies from person to person and does not have objectivity.

Based on this, the author thinks that through learning this sort of inconsistency, a potential degree $g_{x}^{b}$ of preference can be obtained, and the degree of preference of the $\operatorname{tag}^{i}$ to the instance can be experienced in the form of numbers. The specific ideas can be divided into two points: first, to generate a common behavioral preference distribution that can be compatible with multiple inconsistent rankings, second, to learn from the mapping of behavioral preference distribution distributions to instances.

\subsection{Common Preference Construction}

Assume that for a sequence of behaviors $x$, the degree of preference of the tag $i$ for the behavior tag $b$ is expressed as a real number $g_{x, i}^{b}$, and $\sum_{\mathrm{b}} g_{x, i}^{b}=1$.

Defining the degree of preference of the ${ }^{\mathrm{r}_{i}}$ annotator as $B_{\mathrm{i}}, B_{\mathrm{i}}^{\mathrm{b}}=g_{x, i}^{b}, B_{c}$ to indicate the degree of 
common preference, is compatible with the degree of preference of all annotators.

$$
B_{c}=\underset{B}{\arg \min } \sum_{i=1}^{k} D\left(B, B_{i}\right)
$$

In formula (1), $D$ represents two distributed distance functions, and $B_{i}^{b} \geq 0$,the degree of virtual behavior label preference $g_{x, i=0 .}^{v} i=1, \ldots, k, b=0, \ldots, c, \sum_{b=1}^{c} B^{b}+v B^{0}=1 \sum_{b=1}^{c} B_{i}^{b}+v B_{i}^{0}=1$. Assume that behavior labels $\mathbf{l}$ are more appropriate when facing a sequence $x$ of behaviors. So $B_{i}^{j}+\varepsilon \leq B_{i}^{\mathrm{l}}$.

Based on the above formula, this paper chooses the distance $K L$ to describe the similarity between the two fabrics. The specific function formula is:

$$
T\left(B_{c}\right)=\arg \min \sum_{i=1}^{r}\left(\sum_{b=1}^{c} B^{b} 1 n \frac{B_{b}}{B_{i}^{b}}+v B^{0} 1 n \frac{B^{0}}{B_{i}^{0}}\right)
$$

\subsection{Preference Distribution Model Learning}

After obtaining the common preference distribution, a preference distribution model needs to be analyzed. Assume that the given training set is:

$$
\operatorname{Tr}=\left\{\left(x_{1}, B_{1}\right),\left(x_{2}, B_{2}\right), \ldots,\left(x_{n}, B_{n}\right)\right\}
$$

In formula (3), ${ }^{x_{i}}$ is a sequence of behaviors,$B_{i}=\left\{g_{x i}^{b 1}, g_{x i}^{b 2}, \ldots, g_{x i}^{b m}\right\}_{\text {represents the }}$

preference distribution of the sequence of the $i$ behavior. In the learner, the horizontal coordinate represents the behavior label, the vertical coordinate represents the degree of description of the behavior sequence by the behavior label. The distribution of specific preference behavior labels is shown in Figure 1.

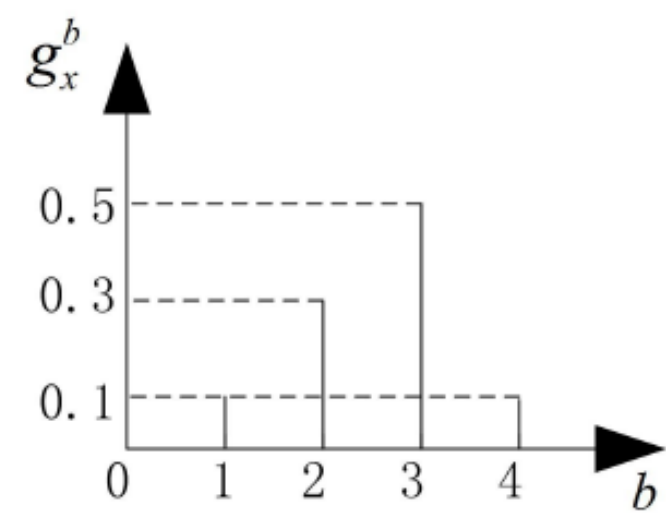

(a)

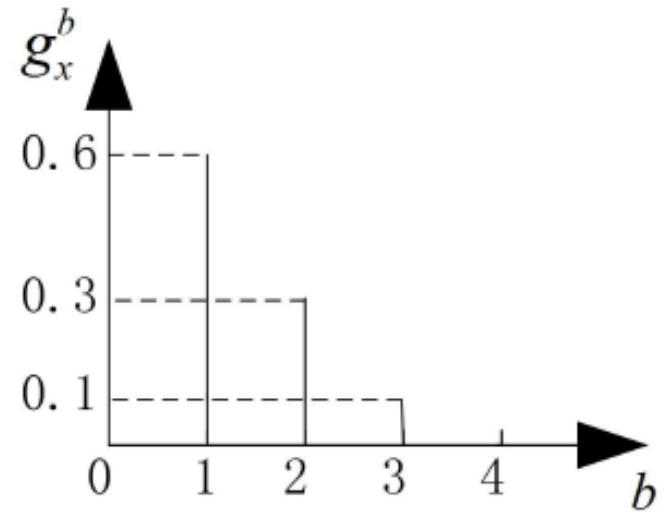

(b)

Figure 1 Preference Behavior Label Distribution

The content shown in the above figure shows that the $\mathrm{g}_{x}^{b}$ is actually a conditional probability distribution $\mathrm{p}(\mathrm{b} / x)$. Thus, the ultimate goal can be seen as a learning of a conditional distribution $\mathrm{p}(\mathrm{b} / x, \theta)$, with parameters to obtain a predictive conditional conditional probability parameter model $\mathrm{p}\left(\mathrm{b}_{\mathrm{j}} / x_{j}, \theta\right)$.

Using the distance $K L$ to describe the similarity between two distributions in the

distribution of preferences, assuming $D\left(P_{\mathrm{i}}^{*} \| P_{i}\right)$ is the distance between the expected distribution $P_{\mathrm{i}}$ and the true distribution $P_{\mathrm{i}}^{*}$, the similarity objective function is: 


$$
\begin{aligned}
T(\theta) & =\underset{\theta}{\arg \min } D\left(P_{i}^{*} \| P_{i}\right) \\
& =\underset{\theta}{\arg \min } \sum_{i} \sum_{j} D\left(g_{x_{i}}^{b_{j}} \| p\left(b_{j} / x_{i} ; \theta\right)\right) \\
& =\underset{\theta}{\arg \min } \sum_{i} \sum_{j}\left(g_{x_{i}}^{b_{j}} 1 n \frac{d_{x_{i}}^{b_{j}}}{p\left(b_{j} / x_{i} ; \theta\right)}\right.
\end{aligned}
$$

among them,

$$
p(b / x ; \theta)=\frac{1}{w} \exp \left(\sum_{i} \theta_{b, t} f_{t}(x)\right)
$$

\subsection{Determine the number of behavior labels}

According to the above model, even if a tag-less behavior sequence is input in the model, the behavioral tags of the sequence can be obtained separately. However, because certain behavior sequences have multiple behavior labels, the model needs to determine the number of behavior labels before acquiring behavior labels. Currently, there are two methods for determining the number of behavioral labels, namely the threshold method and the similarity number method. Among them, the threshold method mainly adopts the method of sorting the description of the behavior label by the behavior label, and determines the number of behavior labels, the similarity number method always holds the concept that the instances have smoothness, that is, the similar behavioral sequences have similar behavioral characteristics and similar number of tags, the number of optimal tags can be determined by finding similar behavioral sequences. These two methods have their own advantages in the application process, however, compared with the similarity method, the threshold method is more suitable for determining the number of complex labels. Therefore, this article will use the threshold method to determine the number of tags.

\section{Crowd Abnormal Behavior Identification Process Design}

In crowd abnormal behavior identification, crowd feature extraction is the key to realize crowd abnormal feature recognition. Specific feature extraction algorithm is used to extract the relevant characteristics of the target group's behavior and habits, so as to provide basis for further identification and classification. Methods for extracting crowd characteristics include multiple methods based on images and objects, methods based on object features, and methods based on image frames.

Feature extraction based on images and objects generally uses edge detection and background/foreground subtraction, and considers only the underlying density estimation information. Researching relevant literature data found that for this kind of feature extraction method, some scholars have proposed to detect the feature changes with the hybrid Gaussian algorithm, and then use the expectation maximization algorithm to obtain the variable values and update probability equation distribution. Some scholars have also proposed that the measured distance of the geometric correction foreground pixel function is the same proportion, and the affine transformation and geometric correction of the pixel count are used for rapid scaling. In addition, some scholars have proposed a method to classify population density features by extracting the number of pixels at the city edge and using optical flow vectors and edge pixels. This article selects the optical flow method to extract image features.

At the same time, after the feature extraction is completed, it is necessary to combine the above algorithms to identify abnormal behaviors and finally complete the abnormality detection. Therefore, combining the above analysis, this article will design the overall crowd abnormality identification process as follows:

1) Combining intelligent video monitoring system, complete the basic collection of crowd images, 
2) Combined with the optical flow method, the abnormal feature extraction of the crowd was completed, which laid the foundation for follow-up crowd abnormality detection,

3) In combination with the preference distribution model, the abnormal population is tagged and the tag is used to determine its abnormal behavior.

4) Output results.

\section{Experimental Results and Analysis}

Since there is no universal data set for analysis of crowd abnormal behavior, the video data set captured by the camera is selected for verification in the algorithm experiment of this paper. Taking into account the diversity of pedestrian movement direction, when shooting video data sets, the stability of the camera should be maintained, and the process of crowd movement should be photographed with the shooting speed of 25 frames/s and the image size of $360 * 240$, and try to ensure that each individual in the crowd occupies an approximate proportion of the image. In addition, the shooting direction should be maintained at about 45 degrees to ensure the consistency of the model input video data. In the algorithm experiment of this paper, the video data set consists of video data of abnormal behavior types such as panic, gang fight, stampede, and fall. The labels of abnormal conditions include normal, stampede, fall, panic, and gang fight.

The selection of features is one of the important factors affecting the classifier's learning results. In the experiment, the improved optical flow features are the characteristics of the crowd behavior descriptors. Specifically, the improved optical flow characteristics are based on Brox's variational optical flow algorithm. Five channels including brightness, green minus red color components, green minus blue color components, and $\mathrm{x}$ gradient and y gradient images are added and preserved grayscale image gradient channels to achieve traffic estimation. At the same time, the smoothing term is modified to reduce the effect of the discontinuous flow problem in the smaller part of the image gradient.

Table 1 Number of training sets and test sets

\begin{tabular}{cccc}
\hline & class & \multicolumn{3}{c}{ sample } \\
\hline Number & Name & Training (frames) & Test (frames) \\
\hline $\mathbf{1}$ & Fall & $\mathbf{7 0}$ & $\mathbf{1 3 0}$ \\
$\mathbf{2}$ & Trample & $\mathbf{7 0}$ & $\mathbf{1 3 0}$ \\
$\mathbf{3}$ & Gang fight & $\mathbf{1 0 0}$ & $\mathbf{2 0 0}$ \\
$\mathbf{4}$ & Panic & $\mathbf{3 0}$ & $\mathbf{7 0}$ \\
$\mathbf{5}$ & Normal & $\mathbf{3 0}$ & $\mathbf{7 0}$ \\
$\mathbf{6}$ & All & $\mathbf{3 0 0}$ & $\mathbf{6 0 0}$ \\
\hline
\end{tabular}




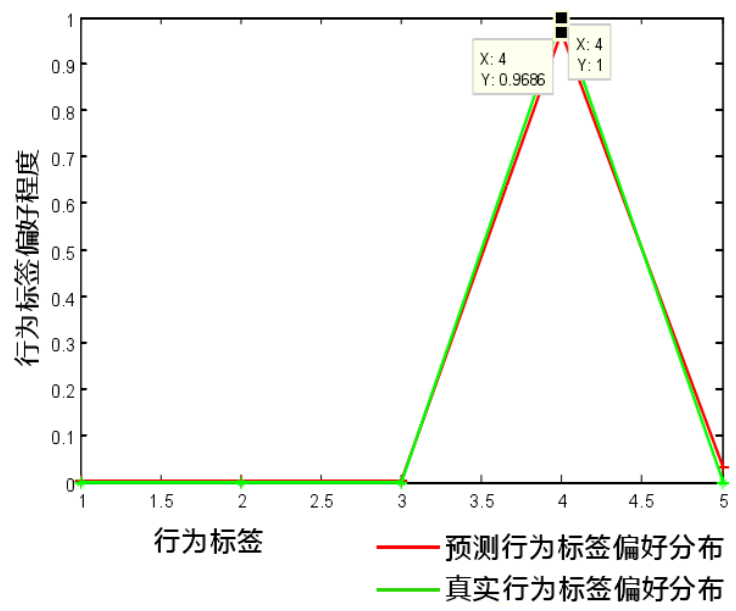

Figure 2 Test results

This paper validates the algorithm based on the training set and the number of test sets shown in Table 1, and also obtains the test set behavior label distribution. The test results of the two samples are shown in Figure 2.

From the test results shown in Figure 2, we can see that the predicted behavior label part is represented by the red line, while the distribution of the real behavior label is represented by the green line, and the abscissa represents the fall, stampede, gang fight, panic, and normal. Tags. The comparison between the two results shows that the proposed algorithm has a good effect. When the behavior description threshold is T3.0, the corresponding label is the label of the behavior sequence. For the test sample 1, the description is the most stepping on the label, and the description of the rest of the labels is below the threshold. Therefore, it can be determined that the abnormality in the test sample 1 is stampede.

\section{Conclusion}

Through the above analysis, we can see that in the detection and identification of traditional abnormal populations, how to use multiple labels to label longer behavior sequences is the focus of thinking in the current year. This paper puts forward a method based on preference distribution on the basis of traditional single labeling, and uses the KL distance similarity calculation method to complete the labeling of behaviors with common similarity. Finally, the experiment verifies the feasibility and correctness of the proposed algorithm, which provides a new channel and method for judging the crowd abnormality in the current intelligent monitoring.

\section{Acknowledgement}

General project of science and technology program of Beijing Education Committee XY-YW02-201602

\section{References}

[1] KaiqiHuang, Xiaoyu Chen, Yunfeng Kang, Tieiu Tan. An Overview of Intelligent Video Surveillance Technology [J]. Chinese Journal of Computers, 2015, v.38, No.39006:1093-1118.

[2] Yongchao Wei, Xia Zhuang, Qiang Fu. Research Progress of Crowd Abnormality Identification Technology [J]. Computer System Application, 2016, v.2509: 10-16.

[3] Haifeng Sang, Wei Chen, Dakuo He. Detection of crowd aggregation and running behavior based on overall characteristics [J]. Optoelectronics· Laser, 2016, v.27, No.24701:52-60.

[4] Yanni Wang, Jun Li, Siming Tian. Abnormal behavior detection based on fuzzy ISODATA clustering and histogram entropy algorithm [J]. Modern Electronic Technology, 2017, v.40, 
No.49112: 120-123+127.

[5] Jian Li, Zhihong Liu, Fei Jiang. An Intelligent Vision-based On-Site Situation Sensing Technology for Mass Events[J]. Ordnance Industry Automation, 2015, v.34, No.20006:61-65.

[6] Zhijun Yao, Juntao Liu, Yu Zhou, Wenyu Liu. Similarity measure method based on symmetric $K L$ distance[J]. Journal of Huazhong University of Science and Technology (Natural Science),2011,39(11):1-4+38.

[7] Yanni Wang, Jun Li, Siming Tian. Detection of Abnormal Behavior of Fuzzy ISODATA Clustering Combined with Histogram Entropy Algorithm [J]. Modern Electronic Technology, 2017, 40(12): 120-123+127.

[8] Zhipeng ZHU, Jianqiang DU, Yingfeng LIU, Fang YU, Jiengen LUO. Calculation of Chinese medicine prescription similarity based on LDA topic model[J]. Journal of Computer Applications, 2017, 34(06): 1668-1670+1676.

[9] Ke Luo, Anzhong Zhou. Face feature extraction model based on convolutional neural network based on KL distance[J]. Journal of Changsha University of Science and Technology (Nature Science), 2017, 14(02): 85-91.

[10] Lian Xu, Ningyan Lu, Yiwen Wang, Xiaomei Chen. Distribution Model of Preference for Gender Information in Faces[J]. Human Ergonomics, 2012, 18(04): 7-11+15. 